\title{
INOVAÇÃO E DESEMPENHO NA ADMINISTRAÇÃO JUDICIAL: DESVENDANDO LACUNAS CONCEITUAIS E METODOLÓGICAS ${ }^{1}$
}

\author{
Marcos de Moraes Sousa \\ Doutorando em Administração pela Universidade de Brasília - UnB \\ Professor do Instituto Federal Goiano - IF Goiano \\ marcos.moraes@ifgoiano.edu.br (Brasil)
}

\section{Tomás de Aquino Guimarães}

Doutor em Sociologia pela Universidade de São Paulo - USP

Professor Titular da Universidade de Brasília - UnB

tomas.aquino.guimaraes@gmail.com (Brasil)

\section{RESUMO}

A inovação - um novo produto ou processo tecnológico, organizacional ou de marketing - tem como pressuposto a melhoria do desempenho. Quando se trata de serviços públicos, notadamente no Judiciário, a inovação configura-se como um fenômeno mais complexo e multifacetado. Neste caso, a literatura acadêmica deveria ser mais bem explorada sob a ótica da inovação. O objetivo do presente ensaio é realizar uma discussão articulada sobre os constructos inovação e desempenho no judiciário, demonstrar suas relações, lacunas conceituais e metodológicas e propor uma agenda de pesquisa. Foi analisado o estado da arte correspondente a esses conceitos, que permitiu classificar os estudos nesses temas em três dimensões: organizacional-gerencial, político-legal e tecnológico. Os resultados indicam que: (i) existem variáveis similares entre os constructos; (ii) a literatura de desempenho é mais volumosa e consolidada; (iii) contribuições importantes para a dimensão organizacional-gerencial foram encontradas nos estudos da corrente denominada direito e economia; (iv) as principais intersecções foram encontradas na dimensão tecnológica, especificamente entre inovação, desempenho e Tecnologias da Informação e Comunicação - TICs. Ao final é apresentada uma agenda de pesquisa visando preencher lacunas identificadas.

Palavras-chave: Inovação; Desempenho; Judiciário; Administração Pública.

\footnotetext{
${ }^{1}$ Os autores agradecem o apoio financeiro da CAPES (bolsa de estudos para o primeiro autor) e do CNPq (bolsa de
} produtividade em pesquisa e financiamento de projeto de pesquisa em Edital Universal para o segundo autor). 


\section{INTRODUÇÃO}

Inovação pressupõe o desenvolvimento de um produto ou processo novo, introdução de melhorias em produtos ou processos existentes, novas formas de comercialização, novos mercados, nova organização ou ainda novas fontes de matéria-prima, que impliquem na aceitação pelo mercado, como sugere Schumpeter (1982, 2008). Esse conceito é bastante amplo para incluir os serviços públicos, setor em que inovações geralmente configuram-se como novos métodos, arranjos estruturais e processos de trabalho, que contribuem para aumentar a eficiência na prestação de um serviço. Nelson e Winter (2005, p. 539) afirmam que "a eficiência pode não ser o único interesse público, mas por certo é amplamente vista como uma característica geral da boa política".

Considera-se que o setor público tem dificuldade de desenvolver métodos específicos de mensuração do desempenho de inovações e que, assim, parte desse desempenho seria imperceptível (Koch, Cunningham, Schwabsky, \& Hauknes, 2006). Uma parcela dessa dificuldade decorre da existência de múltiplos indicadores de desempenho com resultados de difícil quantificação (Røste \& Miles, 2005). Além disso, o setor público é um ambiente menos favorável à inovação, com ações de punição por iniciativas fracassadas mais severas do que por recompensas pela inovação (Borins, 2001). Apesar dessas dificuldades, o setor público, reconhecidamente, cria a estrutura necessária para o desenvolvimento social e econômico, presta serviço básico e fundamental às pessoas, gerencia vultosos recursos e implementa leis que asseguram a democracia e o controle de recursos; dessa forma, espera-se que inovações no setor público tenham impacto positivo no desenvolvimento de uma nação (Hauknes, 2005).

A administração judicial, que compõe o campo mais amplo da Administração Pública, deveria atuar com a mesma eficiência que se espera de outras esferas do setor público, por exemplo, o legislativo ou o executivo (Willoughby, 1929). No Judiciário, a independência, a alta variabilidade das atividades, a especificidade dos casos e a objetividade no processo de decisão dificultam a aplicação de práticas gerenciais fundamentais para o controle dos processos (Pekkanen \& Niemi, 2013). Essas características tornam o processo de avaliação do desempenho do Judiciário mais complexo. Há certo consenso de que o Judiciário poderia ser mais eficiente, eficaz e efetivo (Alves, 2010; $\mathrm{Ng}, 2011$; Pinheiro, 2003; Sadek \& Rogério, 1994; Sadek, 2004). No entanto, como o Judiciário poderia alcançar padrões superiores de desempenho permanece uma questão em aberto.

É reconhecido que o Judiciário brasileiro sofre problemas de morosidade, ineficiência, dificuldade de acesso e custos altos (Pinheiro, 2003; Sadek \& Rogério, 1994; Sadek, 2004). 
Características como maior fechamento à discussão pública, coesão e homogeneidade desse poder dificultam pesquisas nesse universo (Sadek \& Rogério, 1994). No entanto, há iniciativas recentes, por exemplo, do Conselho Nacional de Justiça (CNJ), no sentido de coletar e manter dados relativos ao desempenho do Judiciário, que podem contribuir para o aumento do volume de pesquisas na área e propiciar a criação de conhecimento sobre administração judicial. Nessa linha, este texto tem como objetivo discutir relações entre inovação e desempenho no Judiciário, identificar lacunas conceituais e metodológicas presentes na literatura da área e propor uma agenda de pesquisa capaz de suprir essas lacunas. Este estudo é relevante por ampliar o conhecimento em área carente de pesquisas empíricas e por fornecer subsídios para estudos capazes de gerar novos conhecimentos e propostas de aperfeiçoamento da administração da justiça.

\section{INOVAÇÃO E DESEMPENHO EM SERVIÇOS PÚBLICOS}

O Manual de Oslo sistematizou o conceito de inovação tendo como base Schumpeter (1982) e definiu inovação como a "[...] implementação de um produto (bem ou serviço) novo ou significativamente melhorado, ou um processo, ou um novo método de marketing, ou um novo método organizacional nas práticas de negócios, na organização do local de trabalho ou nas relações externas" (OCDE, 2005, p. 55). Esse manual sugere quatro tipos de inovação: de produto, referente a produtos e serviços inteiramente novos ou com melhorias importantes implementadas aos já existentes; de processo, referente à melhoria em processos de produção e distribuição; organizacional, que envolve o desenvolvimento de práticas gerenciais; de marketing, referente a mudanças no composto de marketing (produto, preço, promoção, colocação).

O conceito de inovação tem evoluído e ampliado a possibilidade de uso do termo, incluindo inovações sociais (Geoff, 2006), inovações organizacionais (Damanpour \& Aravind, 2011), em serviços (Gallouj, 1997) e no setor público. Halvorsen (2005) sugere tipologias específicas de inovação em serviços públicos: inovação de serviço; de processo; administrativa ou organizacional; de sistemas; de concepção, que pode implicar em nova missão, visão, objetivos, estratégias; mudança radical de racionalidade. Inovação no setor público pode ser entendida como "implementação de uma mudança significante no modo como uma organização opera e provê produtos" e compreende "novas ou significantes mudanças nos serviços e bens, processos operacionais, métodos organizacionais, ou no modo como a organização se comunica com os consumidores” (Bloch, 2010, p. 27).

O desempenho em serviços públicos, por seu turno, configura-se como um conceito multifacetado. A eficiência como medida de desempenho do setor público tem sido discutida desde os

Revista de Administração e Inovação, São Paulo, v. 11, n.2, p. 321-344, abr./jun. 2014. 
primeiros estudos sobre o tema. O artigo seminal de Wilson (1887) abre um debate que ainda perdura na administração pública e tem por base a compreensão da administração como um "campo de negócios" separado da política. Para esse autor, o governo deveria ser tratado como um negócio, preocupado com a maximização da eficiência em uma estrutura centralizada e integrada. A equiparação da administração pública aos negócios e o reconhecimento da eficiência como dimensão fundamental para a avaliação de desempenho ressoou por diversos autores da área (Beard, 1966; Cleveland, 1913; Gulick, 1937). Entretanto, a distinção entre política e administração e a eficiência como medida de desempenho fundamental foram criticadas por diversos autores (Denhardt \& Denhardt, 2000; Waldo, 1948).

As principais idiossincrasias do setor público, que o tornam distinto do setor privado, referem-se à multiplicidade e à amplitude dos objetivos, à responsabilidade difusa e à natureza política das atividades (Appleby, 1949), à maior ambiguidade de objetivos, à pluralidade no processo de decisão e à necessidade de controle público (Denhardt, 2012). Isso implica um processo complexo de tomada de decisão e de avaliação de desempenho e caracteriza-se como intensivo em trabalho, descentralizado e matizado (Nelson \& Yates, 1978), com incentivos assimétricos que penalizam o insucesso com mais rigor do que premiam iniciativas bem-sucedidas (Borins, 2001). Outra diferença entre setor público e setor privado são os bens e serviços públicos, caracterizados por alto nível de indivisibilidade e que, consequentemente, dificultam a exclusão de uso por consumidores e tornam o custo marginal de oferta igual azero (Ostrom \& Ostrom, 1971; Pindick \& Rubinfeld, 2002).

Os objetivos de organizações em geral são definidos considerando índices e valores que permitam sua aferição, sendo que, no setor público, dificilmente os objetivos são expressos concretamente, como sugere Simon (1966). O mesmo autor faz uma analogia com a função de produção utilizada na economia e define quatros níveis para a análise da eficiência, como medida de consecução de objetivos. No nível mais amplo, está a aferição de resultados em relação aos objetivos; o segundo nível refere-se à contribuição das atividades administrativas para a consecução dos objetivos; no terceiro nível, analisa-se a eficiência por meio da relação entre recursos e esforço utilizado; por último, no quarto nível, a análise foca o custo monetário.

A eficiência também pode ser entendida por critérios de desempenho objetivos e subjetivos (Pinney, 1966). Esse autor propõe uma sequência de aferição da eficiência, passando primeiramente pelo nível objetivo para, posteriormente, avaliar o nível subjetivo da seguinte forma: aferição da eficiência mecânica (analogia com a máquina), passando pela economia de custo e envolvendo, por último, medidas subjetivas de satisfação. O mesmo autor sugere que a institucionalização da eficiência 
tem dois problemas - a construção de técnicas para a manutenção de certo nível de eficiência e para a melhoria constante.

Para Appleby (1949), não há diferença entre eficiência em organizações públicas e privadas. Na década de 1970, surgiu a corrente teórica denominada Nova Administração Pública, que propõe a adoção de técnicas gerenciais na administração pública com foco na redução de custos e eficiência e que inclui as estratégias de privatizações e terceirizações (Denhardt, 2012). Esse movimento tem por base o modelo racional de administração com a legitimação dos princípios, valores e pressupostos nas questões estruturais, na gestão científica e na eficiência (Denhardt, 2012).

O movimento Americano "reinventando o governo", descrito por Osborne e Gaebler (1993), contribuiu para a corrente da Nova Administração Pública, segundo a qual a aplicação dos princípios de mercado deveria ocorrer na prestação de serviços públicos; assim, o governo, denominado "empreendedor", separa as decisões relacionadas com papéis políticos e reguladores da prestação de serviços. Há similaridades importantes tanto do movimento reinventando o governo quanto da Nova Administração Pública com as teorias de administração pública anteriores, como a adoção de técnicas de gestão do setor privado e a preocupação com a mensuração e o monitoramento do desempenho. Em que medida essas abordagens que procuram aproximar a administração pública da administração de empresas são aplicáveis ao Judiciário é o que se discute a seguir, iniciando-se com o levantamento referente ao estado da arte sobre inovação e desempenho nesse segmento.

\section{ESTADO DA ARTE SOBRE INOVAÇÃO E DESEMPENHO NO JUDICIÁRIO}

O estado da arte sobre inovação e desempenho no Judiciário foi recuperado mediante busca de artigos publicados nas bases de dados Scielo, Science Direct, Gale, Sage, Springer Link, American Psychological Association, Oxford Journals, Wiley, JSTOR, Cambridge Journals, Web of Science e Emerald, disponíveis no Portal de Periódico da CAPES, e na biblioteca Spell. Foi utilizada a palavrachave inovação, simultaneamente com as seguintes palavras, em português e em inglês: setor público; serviço público; judiciário; e tribunal. Para desempenho no Judiciário foram utilizados os termos: desempenho, simultaneamente com: eficiência; produtividade; judiciário; tribunal; e juiz, em português e em inglês. A recuperação dos artigos ocorreu de julho a setembro de 2013. Não foi estabelecido recorte temporal, o que resultou em artigos publicados entre 1975 e 2013.

Os critérios de seleção dos artigos foram: (1) ter relação com o tema inovação e/ou desempenho no Judiciário; (2) tratar de pesquisa empírica; (3) conter os termos definidos para a busca nas palavraschave, título ou resumo; (4) tratar-se de artigo científico revisado por pares. Foram descartados textos 
como ensaios, entrevistas, resenhas e trabalhos publicados em anais de eventos. Três artigos foram excluídos da amostra por tratarem de estudos em Tribunais de Contas. Foram recuperados 78 artigos, dos quais 57 (73\%) referem-se ao desempenho e os demais 21 artigos (27\%) à inovação. Os países mais pesquisados foram Estados Unidos da América com 36 artigos ( 46\%), representando quase a metade dos estudos, Brasil com 12 ( 15\%), seguidos por França (5), Israel (4), Canadá (4) e Índia (3); os outros países tiveram apenas um estudo. Os textos foram classificados, ainda, segundo a principal dimensão de análise do fenômeno explorado (desempenho ou inovação), dimensão esta identificada como organizacional-gerencial, isto é, relacionada com aspectos microeconômicos ou administrativos; político-legal, focada em aspectos jurídicos ou ideológicos; e tecnológica, focada em desenvolvimento ou adoção de tecnologias. Esses resultados estão na Tabela 1.

Tabela 1- Número de artigos sobre desempenho e inovação no Judiciário, segundo as dimensões de análise

\begin{tabular}{|l|c|c|c|c|c|c|}
\hline \multirow{2}{*}{ DIMENSÃO DE ANÁLISE / TEMA } & \multicolumn{2}{|c|}{ DESEMPENHO } & \multicolumn{2}{|c|}{ INOVAÇ̃̃O } & \multicolumn{2}{|c|}{ TOTAL } \\
\cline { 2 - 7 } & $\mathrm{N}$ & $\%$ & $\mathrm{~N}$ & $\%$ & $\mathrm{~N}$ & $\%$ \\
\hline Organizacional-gerencial & 30 & 91 & 03 & 09 & 33 & 100 \\
\hline Político-legal & 23 & 66 & 12 & 34 & 35 & 100 \\
\hline Tecnológica & 04 & 40 & 06 & 60 & 10 & 100 \\
\hline TOTAL & 57 & 73 & 21 & 27 & 78 & 100 \\
\hline
\end{tabular}

Nota. Fonte: dados da pesquisa.

\section{PERSPECTIVAS TEÓRICAS E METODOLÓGICAS}

Dos estudos recuperados, $29(37,2 \%)$ apoiaram-se em teorias de direito, $16(20,5 \%)$ de economia, $11(14,1 \%)$ de direito e economia, $10(12,8 \%)$ da administração pública, $5(6,4 \%)$ de ciência política e três $(3,8 \%)$ de sociologia. O periódico com maior número de publicação foi o International Review of Law and Economics, com 11 (14\%) artigos. Percebe-se que inovação e desempenho no Judiciário são temas ainda pouco explorados pela área da administração; além disso, abordagens que envolvem múltiplas áreas e múltiplas teorias podem contribuir dada a complexidade do tema. Por exemplo, a investigação do fenômeno inovação pode ser abordada pela lente da administração em diferentes níveis de análise tendo por base indicadores e mensurações do direito e da economia.

Um significativo número de estudos com 35 casos, $45 \%$ do total, pesquisou mais de um ramo do direito. Os ramos mais estudados foram: criminal com 14 estudos, civil com cinco, civil e criminal com dois, administrativo e trabalho com três estudos cada. Mais da metade dos estudos, 49 (62,8\%), tiveram como nível de análise o nível organizacional. O nível individual e o ecológico tiveram a mesma frequência de estudos, 14 (17,9\%). Foi identificado apenas um estudo que declarou ter 
utilizado mais de um nível de análise. Quanto à metodologia, os estudos apontam maior frequência de abordagem quantitativa, com 45 textos $(57,7 \%)$, seguidos de abordagem qualitativa com $26(33,3 \%)$ e apenas sete (9\%) utilizaram ambas as abordagens. Quanto à natureza dos dados, houve predominância de dados secundários com 43 artigos $(55,1 \%)$, seguida de pesquisas que utilizaram conjuntamente dados primários e secundários com 18 artigos (23,1\%); somente dados primários foram detectados em 17 estudos (21,8\%). Esses dados são mostrados na Tabela 2.

Tabela 2- Distribuição dos artigos segundo a abordagem metodológica e natureza dos dados

\begin{tabular}{|c|c|c|c|c|c|c|}
\hline & & & & ABORDAGE & & Total \\
\hline & & & $\begin{array}{l}\text { Quali- } \\
\text { quanti }\end{array}$ & Qualitativa & Quantitativa & \\
\hline & & Artigos & 3 & 10 & 4 & 17 \\
\hline & Primárı & $\%$ & 3,8 & 12,8 & 5,1 & 21,8 \\
\hline & Primário e & Artigos & 3 & 10 & 5 & 18 \\
\hline NATUREZA DOS DADOS & secundário & $\%$ & 3,8 & 12,8 & 6,4 & 23,1 \\
\hline & & Artigos & 1 & 6 & 36 & 43 \\
\hline & Secundário & $\%$ & 1,3 & 7,7 & 46,2 & 55,1 \\
\hline & & Artigos & 7 & 26 & 45 & 78 \\
\hline Total & & $\%$ & 9,0 & 33,3 & 57,7 & 100,0 \\
\hline
\end{tabular}

Nota. Fonte: dados da pesquisa.

O recorte longitudinal foi o mais empregado nos estudos $(64,1 \%)$, seguido pelo transversal $(35,9 \%)$. A natureza da amostragem registrou $11(14,1 \%)$ estudos de caso, sendo oito de apenas uma unidade de análise e três com mais de uma unidade de análise; 15 (19,2\%) estudos utilizaram amostra de apenas uma unidade de análise e $52(66,7 \%)$ artigos mais de uma unidade. O delineamento dos estudos apresentou dois experimentos (Goldkamp, 1994; Yunker, 1983) e um quase-experimento (Greene, Sprott, Madon, \& Jung, 2010); os restantes foram estudos correlacionais.

A principal técnica de coleta de dados foi a análise de documentos, presente em 62 estudos, $79,5 \%$ do total. Dezessete estudos utilizaram entrevistas e 13 usaram questionários. Em 11 pesquisas foram utilizadas observações, destacando-se duas etnografias (Fagan \& Malkin, 2002; Hara, 2007), sendo que Hara (2007) utilizou a técnica denominada shadowing - acompanhamento de um sujeito ao longo da execução de seu trabalho e, em alguns casos, até posterior ao trabalho (Czarniawska, 2008) com dois advogados. Os procedimentos de análise dos dados mais utilizados foram: o explicativo com 38 estudos, o descritivo com 32, o exploratório com quatro, somente análise de conteúdo com dois, e somente análise de narrativa com dois. 


\section{RESULTADOS}

\subsection{Inovação no Judiciário}

O primeiro estudo recuperado sobre inovação no Judiciário data de 1994. Percebe-se aumento significativo de publicações sobre o tema nos últimos anos. As principais dimensões referem-se a inovações organizacionais e gerenciais: adoção e melhorias de técnicas de planejamento, acompanhamento e controle da gestão; inovações judiciais, de natureza político-legal, relacionadas principalmente com mudanças legais e de procedimentos de julgamento; e inovações tecnológicas, em especial aquelas centradas na utilização de tecnologias de informação e comunicação em tribunais.

$\mathrm{Na}$ dimensão organizacional-gerencial, foram recuperados três estudos, com a primeira publicação em 2009. Esses estudos trataram da gestão da inovação no Superior Tribunal de Justiça no Brasil (Guimaraes, Odelius, Medeiros, \& Santana, 2011), do programa de ombudsman no Judiciário de Nova Jersey (Bertran, 2001) e do processo colaborativo de tomada de decisão na criação de um comitê local de coordenação de justiça criminal no Estado de Wisconsin (Kempinen, 2009).

O estudo de Guimaraes et al. (2011) analisa a implementação de modelo gerencial de planejamento estratégico e de processo, baseado em tecnologia da informação e em técnicas de gestão de projetos. A inovação contribuiu para a visão mais sistêmica dos processos e atividades do Tribunal bem como para criação de rotinas de planejamento por meio de objetivos e indicadores. No estudo de Bertran (2001), o programa de ombudsman, entendido como inovação, teve boa aceitação e permitiu a participação da comunidade. Kempinen (2009) avaliou inovações adotadas localmente pelas comunidades em parceria com tribunais estaduais e o tribunal superior de Wisconsin, em 2006, e um ano após verificou quais foram abandonadas e quais permaneceram, sendo o processo colaborativo de tomada de decisão o mais significativo.

A dimensão político-legal foi predominante, com 12 estudos, que utilizaram principalmente os conceitos de legitimidade, efetividade, poder, ideologia e liderança. A inovação é considerada principalmente como inovação legal, de métodos e procedimentos jurídicos, acesso ao Judiciário e difusão de inovações jurídicas. Esta dimensão apresentou predominância de estudos qualitativos e contribuições de pesquisadores das áreas de Direito e de Ciência Política. Destaca-se que o país mais pesquisado nessa dimensão foi Estados Unidos da América, com 10 estudos (83\%), sendo um estudo no Brasil $(8 \%)$ e um na China $(8 \%)$.

A inovação na perspectiva político-legal foi compreendida de diferentes formas: combinação de métodos de tratamento de drogas com processamento criminal (Goldkamp, 1994); reformas de

Revista de Administração e Inovação, São Paulo, v. 11, n.2, p. 321-344, abr./jun. 2014. 
procedimentos judiciais (Goodman, Quas, Bulkley, \& Shapiro, 1999); mensurações de ideologias no processo de escolha de juízes para instâncias superiores (Giles, Hettinger, \& Peppers, 2001); métodos de avaliação patrimonial por juízes (Chen, Yee, \& Yoo, 2010); evolução da doutrina de discriminação sexual no ambiente de trabalho (Moyer \& Tankersley, 2012); desenvolvimento e implementação de justiça comunitária como legitimação do Judiciário (Fagan \& Malkin, 2002); criação de tribunais específicos, como o de drogas e de saúde mental, que atendem com consultas e assistência (Trawver \& Rhoades, 2012); difusão de inovações jurídicas (Lutz, 1997); "táticas de inovação", entendidas como a criação de requisitos legais inovadores, ativismo e iniciativa (He, 2013); interação de estabilidade e mudança e a relação entre criação de legislação pelo congresso e aplicação pelos tribunais (Barnes, 2008); e evolução histórica de uma doutrina (Ho \& Ross, 2009). O desempenho das inovações no Judiciário foi avaliado tendo como referência o impacto na carga de trabalho (Goldkamp, 1994) e efetividade percebida (Fagan \& Malkin, 2002; Goodman et al., 1999).

A dimensão tecnológica é relativamente recente em estudos de inovação no Judiciário, tendo sido recuperados seis artigos, sendo que o mais antigo foi publicado em 2003. O processo de inovação nessa dimensão está relacionado, principalmente, com o uso de tecnologias de informação e comunicação - TICs. McKechnie (2003) discute fatores facilitadores do uso da Internet por tribunais da Nova Zelândia. Joia $(2008,2009)$ avaliou resultados intangíveis do projeto Government to Government entre o Banco Central do Brasil e 30 Tribunais de Justiça Estaduais. Neste estudo, a inovação é entendida como um dos componentes do capital intelectual, e o resultado sugere que o projeto impactou positivamente o capital intelectual dos tribunais. Crunkilton (2009) avaliou uma ferramenta computacional de um Tribunal de Drogas no sudeste dos EUA na perspectiva do cliente e dos funcionários do tribunal. $\mathrm{O}$ autor encontrou evidência de que a ferramenta foi percebida como eficiente em termos administrativos e de procedimentos de tratamento ao usuário.

O desenvolvimento e a adoção de processo eletrônico pelo Judiciário Francês foram analisados por Velicogna, Errera e Derlange (2011, 2013). Os autores concluíram que o desenvolvimento do processo eletrônico na justiça é complexo e identificaram os seguintes fatores relevantes de implementação: o compartilhamento de experiências e recursos bem como os contextos tecnológico, organizacional, normativo e institucional.

Os estudos da dimensão tecnológica utilizaram predominantemente a percepção dos usuários, por exemplo, servidores, juízes, advogados, demandantes e demandados da justiça. Apenas um estudo utilizou a abordagem quantitativa, cujas técnicas foram ANOVA e correlação, e também há o predomínio de procedimentos de análise de dados descritivos. A intersecção entre inovação e 
desempenho na dimensão tecnológica ocorreu com a percepção dos usuários do sistema judicial com resultados de eficiência, eficácia, efetividade e accountability.

\subsection{Desempenho no Judiciário}

Quanto ao tema desempenho no Judiciário, foram encontrados estudos mais antigos do que os de inovação, tendo sido selecionados 57 artigos. O primeiro estudo foi publicado em 1975, também mostrando aumento significativo nos últimos anos.

A dimensão organizacional-gerencial descreve processos de mensuração de indicadores judiciais e gestão do desempenho organizacional. Destaca-se entre os estudos dessa dimensão uma tradição teórica denominada direito e economia, cuja origem é creditada a Landes (1971) e a Posner (1973). Essa dimensão foi predominante nos estudos de desempenho, com 30 artigos selecionados, contendo um estudo de 1975, o mais antigo de toda a amostra. Este conjunto de artigos apresenta diferentes matizes epistemológicos e metodológicos. Os assuntos abordados incluem alocação de recursos, aspectos administrativos, eficiência e qualidade judicial.

O primeiro estudo (Gillespie, 1975) analisou a alocação de recursos para atendimento da demanda de serviços judiciais e utilizou, como uma das principais variáveis, o tempo do juiz dedicado à atividade jurisdicional. Segundo o autor, os tribunais têm uma incumbência social para atender várias demandas por serviços judiciais e, portanto, os recursos alocados devem ser suficientes para atender a demanda. Leone e Kinkade (1993) procuraram determinar, sob a lente da teoria institucional e de sistemas, como a estrutura e as operações de tribunais criminais da Califórnia mudam conforme o resultado de aumentos orçamentários e, consequentemente, verificaram se o desembolso resulta em aumento na taxa de processamento de causas. Os autores concluem que os tribunais de primeira instância têm maiores aumentos de produtividade em relação ao aumento orçamentário do que os tribunais de instâncias superiores.

A análise dos aspectos administrativos no Judiciário teve como interesse os tipos e as fontes de conflito no desempenho do trabalho de gestores de tribunais (Mays \& Taggart, 1986), gestão de desempenho (Pekkanen \& Niemi, 2013; Schneider, 2004), parcerias entre tribunais e outras organizações (Kent, 2005), análise de melhores práticas de gestão (Binford, Greene, Schmidlkofer, Wilsey, \& Taylor, 2007), accountability judicial e gerencial (Contini \& Mohr, 2007), percepção de cultura (Matz, Adams, \& Williamson, 2011), métodos de resolução de conflitos (Kovač, 2013) e gestão da imagem $(\mathrm{Wu}, 2013)$.

Revista de Administração e Inovação, São Paulo, v. 11, n.2, p. 321-344, abr./jun. 2014. 
Os estudos que avaliam especificamente a eficiência tiveram início com o trabalho de Lewin, Morey e Cook (1982). Esses autores utilizaram a técnica denominada Análise Envoltória de Dados (DEA - Data Envelopment Analysis) para a comparação da eficiência entre 100 tribunais criminais superiores, na Carolina do Norte. As variáveis de desempenho foram casos julgados por ano e número de casos pendentes, com prazo maior do que noventa dias. Os autores concluem que a DEA pode ser útil na avaliação da eficiência de tribunais.

Kittelsen e Førsund (1992) utilizaram a DEA para examinar a eficiência de tribunais distritais na Noruega, no período de 1983 a 1988, com o intuito de sugerir formas de melhorar a eficiência e analisar métodos aplicáveis ao setor público em geral. Pedraja-Chaparro e Salinas-Jimenez (1996) mensuraram a eficiência de tribunais da Espanha também por meio de DEA e por regressão TOBIT. Smyth e Bhattacharia (2003) examinaram o relacionamento entre envelhecimento do juiz e produtividade no Tribunal Federal Australiano por meio de estudo inferencial.

Beenstock e Haitovsky (2004), por meio de dados em painel, avaliaram o efeito do número de juízes no número de casos resolvidos em 25 tribunais israelenses em três instâncias, no período de 1964 a 1995. As variáveis independentes foram casos distribuídos, casos pendentes e número de juízes. Os autores concluem que o aumento da pressão da carga de trabalho impacta positivamente o desempenho e não há evidência de que o aumento no número de juízes tenha efeito positivo sobre o desempenho. Schneider (2005) examinou como o sistema de tribunais do trabalho Alemão configurou o desempenho do tribunal no período de 1980 a 1998, também por meio de DEA.

O estudo de Costa, Martinewski, Vieira, Michel e Michel (2006) identificou os seguintes indicadores de carga de trabalho de magistrados: número de processo por classe processual, funções exercidas, total de horas semanais em atividades relacionadas ao processo judicial e em atividades administrativas, total de horas anuais em atividades de representação e em atividades educacionais e científicas, total de horas mensais em outras atividades, número de processos recebidos mensalmente pelo juiz, disponibilidade de pessoal. O estudo conclui que magistrados trabalham em média 54 horas semanais diretamente relacionadas à magistratura.

Mitsopoulos e Pelagidis (2007) avaliaram por meio de dados em painel o tempo gasto pelo tribunal para concluir um caso na Grécia, em tribunais de primeira instância e de recursos, e identificaram: (i) crescimento constante no tempo gasto para conclusão de casos, (ii) que a razão entre número de funcionários e número de casos afeta o tempo em tribunais de recursos e tribunais superiores, entretanto, não em tribunais administrativos de primeira instância.

Rosales-López (2008) avaliou o desempenho de 61 tribunais de Andaluzia, na Espanha, por meio da abordagem econômica, com o objetivo de identificar se a baixa taxa de recurso nas decisões e alta

Revista de Administração e Inovação, São Paulo, v. 11, n.2, p. 321-344, abr./jun. 2014. 
taxa de output (sentenças e decisões) são metas incompatíveis no Judiciário. A autora conclui que: (a) os tribunais podem produzir mais com os recursos atuais; (b) as variáveis independentes tiveram coeficientes de regressão positivos com desempenho, com exceção de rotatividade de juízes; (c) alcançar alta produção e baixa taxa de recursos não são objetivos incompatíveis.

Abramo (2010) analisou e propôs método de mensuração de desempenho de tempo médio de espera de decisões para o Supremo Tribunal Federal do Brasil. A avaliação pode ser comparada por ministro, por ramo e classes processuais. $\mathrm{O}$ autor vislumbra a possibilidade de a análise dos tempos médios fomentar a adoção de medidas gerenciais que melhorem a celeridade das decisões e que também sirvam como incentivo à reputação.

Deyneli (2012) procurou identificar, por meio de DEA em dois estágios e regressão TOBIT, o relacionamento entre eficiência dos serviços da justiça e os salários de juízes em países Europeus. O salário do juiz foi positivamente significante para a eficiência dos tribunais; todavia, o autor alerta que essa não deve ser enxergada como única solução para a melhoria da eficiência dos serviços do Judiciário.

Yeung e Azevedo (2011) utilizaram a técnica DEA em uma amostra de 27 tribunais estaduais brasileiros para mensurar a eficiência, entendida como a relação entre insumos (número de juízes e pessoal de apoio ponderado pela carga de trabalho) e produto (casos resolvidos em primeira e segunda instância), e criaram um índice de qualidade organizacional dos tribunais estaduais brasileiros. O estudo encontrou evidência de que a eficiência varia significativamente entre os tribunais; a falta de recursos humanos ou materiais não pode ser considerada como o principal fator de ineficiência; e a qualidade organizacional da gestão tem impacto positivo na eficiência do tribunal.

Christensen e Szmer (2012) examinaram causas de ineficiência em decisões por meio de dados em painel, em 13 tribunais de apelações americanos, de 1971 a 1996. A variável dependente foi o número de dias para a decisão de um caso. Os maiores efeitos foram relacionados com mecanismos institucionais, como férias, número de juízes, número de juízes por milhas quadradas e a técnica de decisão por argumentação oral. Dimitrova-Grajzl, Grajzl, Sustersic e Zajc (2012) examinaram um painel de tribunais de primeira instância ao longo de nove anos na Eslovênia. A demanda foi um elemento importante para o desempenho judicial e, ceteris paribus, o número de juízes em atuação não afeta estatisticamente o desempenho.

Ramseyer (2012) avaliou determinantes de produtividade judicial e celeridade por meio de decisões de juízes japoneses, publicadas entre 1995 e 2004. A medida de produtividade teve correlação com habilidade (estudar em universidades seletivas) e esforço (escolhido para carreira de elite). O 
autor identificou que celeridade teve correlação fraca com habilidade, esforço e experiência no nível do tribunal.

Buscaglia e Ulen (1997) identificaram, por meio de entrevistas com juízes e análise de documentos, as causas de ineficiência no Judiciário (direito comercial) na América Latina e os meios de corrigi-las. Os autores concluem que aumento de recurso judicial não tem efeito significativo na redução do tempo do processo. Também argumentam que a padronização de procedimento, melhorias na gestão de processos, maior disponibilidade de tecnologia computacional e o tamanho da empresa do demandante são fatores positivamente associados com o desempenho no tempo observado em litígios comerciais na Argentina e Venezuela.

Tulkens (1993) analisou a eficiência técnica em três organizações públicas: banco, tribunal e transporte coletivo. Especificamente sobre o tribunal, avaliou a eficiência na prestação de serviços judiciais. O autor criou um índice de eficiência e comparou a DEA com FDH (Free Disposal Hull).

Anderson IV (2011) avaliou desempenho e qualidade judicial e entendeu esta última como o número de citações de decisões, utilizando uma amostra de 383 juízes federais de tribunal de apelação americano, no período entre 1960 e 2008 e criou um ranking de citações. Choi, Gulati e Posner (2013) analisaram a relação entre habilidade do juiz e qualidade e desempenho judicial; a qualidade judicial foi entendida como frequência de citações e citações positivas.

A dimensão político-legal teve uma amostra de 23 estudos sobre desempenho no Judiciário, com foco no processo de tomada de decisão judicial, no impacto de aspectos políticos e ideológicos e aspectos de reformas mais amplas no Judiciário. As definições tratadas foram: legitimidade, ideologia, poder e efetividade.

Os estudos que investigaram o processo de tomada de decisão judicial avaliaram o desempenho de procedimentos e os métodos de decisão judicial (Myers, 1981; Yunker, 1983), a relação entre ideologia e decisão de juízes do Tribunal Superior dos Estados Unidos da América (Kearney \& Sheehan, 1992; McGuire, Vanberg, Smith Jr., \& Caldeira, 2009), métodos de tomada de decisão comparativamente entre EUA e Austrália (Waye, 2003), o efeito do número de juízes na precisão de decisões judiciais pela comparação dos resultados de recursos ao Tribunal Superior de Israel por dois métodos (casos tratados por um juiz e por três juízes) (Karotkin, 1994), a relação entre restrições do comportamento dos poderes executivo e legislativo e a influência nas decisões do Tribunal de Justiça Europeu (Carrubba, Gabel, \& Hankla, 2008), participação de membros que não pertencem ao Judiciário (julgamento por júri e participação de assessores) na tomada de decisão judicial em 80 países (Voigt, 2009). 
Outros artigos analisaram aspectos políticos, como o impacto da implantação e colapso do autoritarismo no desempenho de funções processuais e administrativas do tribunal superior das Filipinas (Tate \& Haynie, 1993), o nível de apoio da população americana e a mudança de tal apoio ao longo do tempo ao Tribunal Superior (Swanson, 2007), o comportamento de juízes ingleses e aspectos do processo de decisão que podem ser influenciados por pressões políticas (Vidal \& Leaver, 2011).

Os estudos que pesquisaram reformas no Judiciário tiveram como preocupação avaliar o impacto do Judiciário no empreendedorismo (Chemin, 2009a), o efeito da legislação e o processo trabalhista no emprego formal e informal na Índia (Fagernäs, 2010), a experiência do Banco de Desenvolvimento Asiático na implementação de reforma judicial na Ásia (Armytage, 2011), o efeito do número de tribunais superiores na qualidade do sistema legal de um país e do desenvolvimento econômico (Voigt, 2012), o impacto da celeridade do Judiciário na atividade econômica da Índia, por meio das emendas ao Código Civil, que acrescentam ou complicam os procedimentos e por emendas que produzem ambiguidade nos procedimentos (Chemin, 2009a), efeitos da complexidade normativa do sistema legal em atrasos na justiça (Di Vita, 2012).

A intersecção de inovação na dimensão político-legal do conjunto de estudos sobre desempenho no Judiciário foi compreendida como a criação de tribunais comunitários, considerados como inovação; tais tribunais resultaram em melhor acesso ao Judiciário (Gilling \& Jolley, 2012) e na criação de tribunais específicos como o tribunal de drogas (Sevigny, Pollack, \& Reuter, 2013).

A dimensão tecnológica envolve estudos relacionados com adoção de tecnologias por tribunais, principalmente TIC's, e o impacto no desempenho judicial. A amostra contemplou quatro artigos; a natureza amostral de todas as pesquisas foi estudo de caso com abordagem qualitativa; percebe-se a ausência de modelo explicativo de relações causais de desempenho tecnológico no Judiciário.

Daschbach e Swain (1977) estudaram o desenvolvimento de uma base de dados eletrônica de informações do Judiciário de Indiana, EUA. A pesquisa, conduzida no final da década de 1970, já previa a necessidade do uso de TIC's no Judiciário, afirmando que o computador tinha se tornado vital para mensuração da produtividade no Judiciário e, que naquele período, doze Estados Norte Americanos e grande número de condados e municípios tinham iniciado a implementação de banco de dados no sistema judicial. Os autores concluem que empregados com competências adequadas são necessários para colocar em prática o sistema, que tal tecnologia poderia diminuir o crescente uso de papel, que as principais funções eram a inclusão e leitura de dados, criação de novos registros, atualização de arquivos existentes e compilação de relatórios de cargas de trabalho para os tribunais.

Hara (2007) investigou o papel de tecnologias de informação como suporte para práticas profissionais, entendidas como eficiência no trabalho e formação de identidade, por meio de estudo de 
caso etnográfico aplicado em uma defensoria pública norte-americana. A tecnologia foi eficaz no compartilhamento de informações profissionais técnicas e limitada no compartilhamento de significado cultural e na formação de identidade.

Rosa, Teixeira e Pinto (2013) analisaram fatores de risco no projeto, desenvolvimento e na implementação de sistemas de informação de justiça eletrônica, por meio de estudos de casos, comparando Brasil, Portugal, Singapura, Bélgica e Cabo Verde. Saman e Haider (2013) apresentam a adoção e o uso de tecnologia em um tribunal da Malásia e também propõem uma estrutura analítica de gestão eletrônica de tribunais baseada na teoria de sistemas de informação.

\section{LACUNAS, INTERSECÇÕES DOS ESTUDOS EMPÍRICOS E AGENDA DE PESQUISA}

Percebe-se que os estudos empíricos de inovação e desempenho no Judiciário incluem abordagens conceituais e metodológicas distintas, o que caracteriza a área como multidisciplinar, multifacetada e complexa. Os estudos sobre desempenho são mais consolidados e mais antigos do que os de inovação. Foi possível classificar os estudos de ambos os constructos nas dimensões organizacional-gerencial, político-legal e tecnológica. Algumas intersecções importantes ocorreram: (i) algumas variáveis são comuns nos estudos de inovação e de desempenho, como carga de trabalho, tempo gasto na prestação de serviço e efetividade do Judiciário; (ii) a dimensão organizacionalgerencial nos estudos em desempenho está mais consolidada, utiliza técnicas distintas, e elementos da teoria da administração pública são aplicados em ambos os constructos, como a mensuração e ênfase nos resultados, controle e uso de técnicas próprias do setor privado; (iii) a dimensão político-legal tratou de categorias de análise similares tanto no tema inovação como no tema desempenho, por exemplo, ideologia, legitimidade, efetividade e poder; (iv) na dimensão tecnológica, tanto no tema inovação como no tema desempenho têm sido pesquisadas as TIC's, por meio de estudos de percepção de sujeitos envolvidos e estudo de caso.

Alguns caminhos ainda precisam ser trilhados nos temas estudados. Os estudos em inovação poderiam privilegiar também uma orientação mais quantitativa e explicativa, dado que os estudos recuperados predominantemente avaliaram a percepção de sujeitos por meio de estudos exploratórios e descritivos. Os estudos que avaliam a dimensão organizacional-gerencial carecem de análise de inovações, especificamente, estudos que avaliam a eficiência e que poderiam incluir a análise de resultados da adoção de novas tecnologias e práticas gerenciais, proposta também sugerida por Lewin et al. (1982).

Revista de Administração e Inovação, São Paulo, v. 11, n.2, p. 321-344, abr./jun. 2014. 
Ainda, sobre os estudos que avaliam a eficiência, percebe-se o privilégio de variáveis econômicas. A inclusão de variáveis gerenciais poderia revelar aspectos não observados; além disso, a desagregação dos dados, por exemplo, no nível das varas em vez do tribunal e do tempo de duração processual, também poderia revelar padrões de eficiência não observados em níveis mais agregados.

A análise de TIC's, intersecção importante em inovação e desempenho no Judiciário, ainda carece do desenvolvimento de modelos explicativos de relações causais entre a adoção dessas tecnologias e o desempenho. Essas relações possibilitariam a replicação e a comparação dos estudos, visto que apenas seis estudos em inovação e quatro em desempenho abordaram a dimensão tecnológica, e verifica-se a predominância de estudos descritivos e exploratórios.

À luz da análise dos estudos e de lacunas de pesquisa encontradas, sugerem-se alternativas de pesquisa sobre inovação e desempenho aplicado ao Judiciário: (a) estudos que incluam variáveis antecedentes e consequentes da inovação no Judiciário, visto que ainda não há precisão e acordo dos conceitos envolvidos bem como não há replicação dos estudos; (b) pesquisas que objetivem a mensuração da inovação no Judiciário por medidas nomotéticas, visto que há uma grande utilização de percepção dos sujeitos o que pode incorrer em vieses de autorrelato; (c) estudos de mensuração de inovação no Judiciário que utilizem modelos já validados de desempenho; (d) a utilização de elementos multimetodológicos e multiteóricos, principalmente com o envolvimento de teorias organizacionais e da administração pública juntamente com referências do direito e da economia. Essas propostas podem diminuir vieses de estudo com método único já verificado anteriormente; (e) estudos específicos de algumas áreas judiciais, como comercial, trabalho, entre outras, de modo a identificar diferenças e semelhanças interáreas; (f) a utilização de distintos níveis de análise, visto que foi encontrado apenas um estudo com investigação em mais de um nível; (g) estudos que explorem abordagens ao mesmo tempo qualitativas e quantitativas; (h) estudos de mensuração da inovação no Brasil que explorem o potencial dos dados disponibilizados pelo $\mathrm{CNJ}$ e que ainda são pouco explorados, principalmente em estudos de inovação; (i) estudos explicativos de inovação, dada a limitação de generalização de estudos descritivos e exploratórios. 


\section{REFERÊNCIAS}

Abramo, C. W. (2010). Tempos de espera no Supremo Tribunal Federal. Revista Direito GV, 6(2), $423-441$.

Alves, F. L. R. (2010). Processo eletrônico no juizado especial e a ampliação do acesso à justiça. Retrieved October $\quad 18, \quad 2010, \quad$ from http/www.jfce.gov.br/internet/esmafe/.../paperDrFranciscoLuisRios.pdf

Anderson IV, R. (2011). Distinguishing judges: An empirical ranking of judicial quality in the United States Court of Appeals. Missouri Law Review, 76(2), 315-348.

Appleby, P. H. (1949). Big democracy. New York: Alfred A. Knopf.

Armytage, L. (2011). Judicial reform in Asia case study of ADB's experience: 1990-2007. Hague Journal on The Rule of Law, 3(1), 70-105.

Barnes, J. (2008). Courts and the puzzle of institutional stability and change: Administrative drift and judicial innovation in the case of asbestos. Political Research Quarterly, 61(4), 636-648.

Beard, C. A. (1966). Filosofia, ciência e arte da administração. In D. Waldo (Ed.), Problemas $e$ aspectos da administração pública (pp. 97-102). São Paulo: Pioneira.

Beenstock, M., \& Haitovsky, Y. (2004). Does the appointment of judges increase the output of the judiciary? International Review of Law \& Economics, 24(3), 351-369.

Bertran, M. (2001). Judiciary ombusman: Solving problems in the courts. Fordham Urban Law Journal, 29, 2099-2116.

Binford, W. W., Greene, P. C., Schmidlkofer, M. C., Wilsey, R. M., \& Taylor, H. A. (2007). Seeking best practices among intermediate courts of appeal: A nascent journey. The Journal of Appellate Practice and Process, 9(1), 37-119.

Bloch, C. (2010). Copenhagen Manual: Towards a conceptual framework for measuring public sector innovation. Copenhagen: MEPIN.

Borins, S. (2001). Encouraging innovation in the public sector. Journal of Intellectual Capital, 2(3), 310-319.

Buscaglia, E., \& Ulen, T. (1997). A quantitative assessment of the efficiency of the judicial sector in Latin America. International Review of Law \& Economics, 17(2), 275-291.

Carrubba, C. J., Gabel, M. G., \& Hankla, C. (2008). Judicial behavior under political constraints: Evidence from the European Court of Justice. American Political Science Review, 102(4), 435452.

Chemin, M. (2009a). Do judiciaries matter for development? Evidence from India. Journal of Comparative Economics, 37(2), 230-250.

Chemin, M. (2009b). The impact of the judiciary on entrepreneurship: Evaluation of Pakistan's "access to justice programme." Journal of Public Economics, 93(1), 114-125.

Revista de Administração e Inovação, São Paulo, v. 11, n.2, p. 321-344, abr./jun. 2014. 
Chen, F., Yee, K. K., \& Yoo, Y. K. (2010). Robustness of judicial decisions to valuation-method innovation: An exploratory empirical study. Journal of Business Finance \& Accounting, 37(9), $1094-1114$.

Choi, S. J., Gulati, M., \& Posner, E. A. (2013). How well do measures of judicial ability predict judicial performance?: A case study using securities class actions. International Review of Law \& Economics, 33, 37-53.

Christensen, R. K., \& Szmer, J. (2012). Examining the efficiency of the U. S. courts of appeals: Pathologies and prescriptions. International Review of Law \& Economics, 32(1), 30-37.

Cleveland, F. A. (1913). Organized democracy: an introduction to the study of American politics. New York: Longmans, Green, and Co.

Contini, F., \& Mohr, R. (2007). Reconciling independence and accountability in judicial systems. Utrecht Law Review, 3(2), 26-43.

Costa, S. G. da, Martinewski, C. L., Vieira, L. J. M., Michel, N., \& Michel, R. (2006). Mensuração da carga de trabalho de magistrados: Uma análise comparativa do estudo realizado no Tribunal de Justiça do Estado do Rio Grande do Sul com experiências internacionais. Revista Da Faculdade de Direito Da Universidade Federal Do Rio Grande Do Sul, 26(221-255), 1-30.

Crunkilton, D. D. (2009). Staff and client perspectives on the journey mapping online evaluation tool in a drug court program. Evaluation and Program Planning, 32(2), 119-128.

Czarniawska, B. (2008). Organizing: How to study it and how to write about it. Qualitative Research in Organizations and Management: An International Journal, 3(1), 4-20. doi:10.1108/17465640810870364

Damanpour, F., \& Aravind, D. (2011). Managerial innovation: Conceptions, processes, and antecedents. Management and Organization Review, 8(2), 423-454.

Daschbach, J. M., \& Swain, J. J. (1977). The computer in measuring judicial productivity. Computers \& Industrial Engineering, 1(4), 263-271.

Denhardt, R. B. (2012). Teorias da administração pública. São Paulo: Cengage Learning.

Denhardt, R. B., \& Denhardt, J. V. (2000). The new public service: Serving rather than steering. Public Administration Review, 60(6), 549-559.

Deyneli, F. (2012). Analysis of relationship between efficiency of justice services and salaries of judges with two-stage DEA method. European Journal of Law and Economics, 34(3), 477-493.

Di Vita, G. (2012). Normative complexity and the length of administrative disputes: Evidence from Italian regions. European Journal of Law and Economics, 34(1), 197-213.

Dimitrova-Grajzl, V., Grajzl, P., Sustersic, J., \& Zajc, K. (2012). Court output, judicial staffing, and the demand for court services: Evidence from Slovenian courts of first instance. International Review of Law \& Economics, 32(1), 19-29.

Fagan, J., \& Malkin, V. (2002). Theorizing community justice through community courts. Fordham Urban Law Journal, 30, 897-953.

Revista de Administração e Inovação, São Paulo, v. 11, n.2, p. 321-344, abr./jun. 2014. 
Fagernäs, S. (2010). Labor law, judicial efficiency, and informal employment in India. Journal of Empirical Legal Studies, 7(2), 282-321.

Gallouj, F. (1997). Towards a neo-Schumpeterian theory of innovation in services? Science and Public Policy, 24(6), 405-420.

Geoff, M. (2006). The process of social innovation. Innovations: Technology, Governance, Globalization, 1(2), 145-162.

Giles, M. W., Hettinger, V. A., \& Peppers, T. (2001). Picking federal judges: A note on policy and partisan selection agendas. Political Research Quarterly, 54(3), 623-641.

Gillespie, R. W. (1975). An analysis of the allocation of judicial resources: The Illinois experience. Journal of Criminal Justice, 3(3), 207-216.

Gilling, D., \& Jolley, M. (2012). A case suty of an English community court. British Journal of Community Justice, 10(2), 55-69.

Goldkamp, J. S. (1994). Miami's treatment drug court for felony defendants: Some implications of assessment findings. Prison Journal, 74(2), 110-166.

Goodman, G. S., Quas, J. A., Bulkley, J., \& Shapiro, C. (1999). Innovations for child witnesses: A national survey. Psychology, Public Policy, and Law, 5(2), 255-281.

Greene, C., Sprott, J. B., Madon, N. S., \& Jung, M. (2010). Punishing processes in Youth Court: Procedural justice, court atmosphere and youth's views of the legitimacy of the justice system. Canadian Journal of Criminology and Criminal Justice/La Revue Canadienne de Criminologie et de Justice Pénale, 52(5), 527-544.

Guimaraes, T. A., Odelius, C. C., Medeiros, J. J., \& Santana, J. A. V. (2011). Management innovation at the Brazilian Superior Tribunal of Justice. The American Review of Public Administration, 41(3), 297-302.

Gulick, L. (1937). Notes on the theory of organizaion. In L. Gulick \& L. Urwick (Eds.), Papers on the science of administration. New York: Institute of Public Administration Columbia University.

Halvorsen, T. (2005). On innovation in the Public Sector. Oslo: Publin/NIFU/STEP.

Hara, N. (2007). Information technology support for communities of practice: How public defenders learn about winning and losing in court. Journal of The American Society for Information Science and Technology, 58(1), 76-87.

Hauknes, J. (2005). Some thoughts about innovation in the public and private sector compared. Oslo: Publin/NIFU/STEP.

He, X. (2013). Judicial innovation and local politics: Judicialization of administrative governance in East China. The China Journal, 69, 20-42.

Ho, D. E., \& Ross, E. L. (2009). Did liberal justices invent the standing doctrine? An empirical study of the evolution of standing, 1921-2006. Stanford Law Review, 62(3), 591-667. 
Joia, L. A. (2008). The impact of government-to-government endeavors on the intellectual capital of public organizations. Government Information Quarterly, 25(2), 256-277.

Joia, L. A. (2009). Governo eletrônico e capital intelectual nas organizações públicas. Revista de Administração Pública, 43(6), 1379-1405.

Karotkin, D. (1994). Effect of the size of the bench on the correctness of court judgments: The case of Israel. International Review of Law \& Economics, 14(3), 371-375.

Kearney, R. C., \& Sheehan, R. S. (1992). Supreme Court decision making: The impact of court composition on state and local government litigation. The Journal of Politics, 54(4), 1008-1025.

Kempinen, B. (2009). Criminal justice innovations in Wisconsin: Collaborative decision making. The Justice System Journal, 30(3), 327-346.

Kent, J. C. (2005). Getting the best of both worlds: Making partnerships between court and community ADR programs exemplary. Conflict Resolution Quarterly, 23(1), 71-85.

Kittelsen, S. A. C., \& Førsund, F. R. (1992). Efficiency analysis of Norwegian district courts. Journal of Productivity Analysis, 3(3), 277-306.

Koch, P., Cunningham, P., Schwabsky, N., \& Hauknes, J. (2006). Summary and policy recommendations. Oslo: Publin/NIFU/STEP.

Kovač, P. (2013). Effective adjudication through administrative appeals in Slovenia. Utrecht Law Review, 9(3), 39-50.

Landes, W. (1971). An economic analysis of courts. Journal of Law and Economics, 14(1), 61-107.

Leone, M. C., \& Kinkade, P. T. (1993). Bang for the buck: California court processing and budget allocations: 1976-1986. The Social Science Journal, 30(1), 99-111.

Lewin, A. Y., Morey, R. C., \& Cook, T. J. (1982). Evaluating the administrative efficiency of courts. Omega The International Journal of Management Science, 10(4), 401-411.

Lutz, J. M. (1997). Regional leaders in the diffusion of tort innovations among the American states. Publius: The Journal of Federalism, 27(1), 39-58.

Matz, A. K., Adams, J. H., \& Williamson, D. (2011). Understanding culture in Kentucky's courtrooms: An essential tool for management and long-range planning. Juvenile and Family Court Journal, 62(3), 25-42.

Mays, G. L., \& Taggart, W. A. (1986). Court clerks, court administrators, and judges: Conflict in managing the courts. Journal of Criminal Justice, 14(1), 1-7.

McKechnie, D. (2003). The use of the internet by courts and the judiciary: Findings from a study trip and supplementary research. International Journal of Law and Information Technology, 11(2), 109-148.

Mitsopoulos, M., \& Pelagidis, T. (2007). Does staffing affect the time to dispose cases in Greek courts? International Review of Law \& Economics, 27(2), 219-244.

Revista de Administração e Inovação, São Paulo, v. 11, n.2, p. 321-344, abr./jun. 2014. 
Moyer, L. P., \& Tankersley, H. (2012). Judicial innovation and sexual harassment doctrine in the U. S. Courts of Appeals. Political Research Quarterly, 65(4), 784-798.

Myers, M. A. (1981). Judges, juries, and the decision to convict. Journal of Criminal Justice, 9(4), 289-303.

Nelson, R. R., \& Winter, S. G. (2005). Uma teoria evolucionária da mudança econômica. Campinas: Editora da Unicamp.

Nelson, Richard, R., \& Yates, D. (1978). Innovation and implementation in public organizations. Lexingon: D. C. Heath and Company.

Ng, G. Y. (2011). A discipline of judicial governance? Utrecht Law Review, 7(1), 102-116.

OCDE. (2005). Manual de Oslo: Diretrizes para coleta e interpretação de dados sobre inovação (3rd ed.). Brasília: OCDE/FINEP.

Osborne, D., \& Gaebler, T. (1993). Reinventing government: How the entrepreneurial spirit is transforming the public sector. New York: Plume.

Ostrom, V., \& Ostrom, E. (1971). Public choice: A different approach to the study of public administration. Public Administration Review, 31(2), 203-216.

Pedraja-Chaparro, F., \& Salinas-Jimenez, J. (1996). An assessment of the efficiency of Spanish Courts using DEA. Applied Economics, 28(11), 1391-1403.

Pekkanen, P., \& Niemi, P. (2013). Process performance improvement in justice organizations-Pitfalls of performance measurement. International Journal of Production Economics, 143(2), 605-611.

Pindick, R. S., \& Rubinfeld, D. L. (2002). Microeconomia (5th ed.). São Paulo: Prentice Hall.

Pinheiro, A. C. (2003). Direito e economia num mundo globalizado: Cooperação ou confronto? (pp. 1-25). Rio de Janeiro: IPEA. Retrieved http://www.ipea.gov.br/portal/images/stories/PDFs/TDs/td_0963.pdf

Pinney, H. (1966). Níveis e medição de eficiência. In D. Waldo (Ed.), Problemas e aspectos da administração pública (pp. 531-537). São Paulo: Pioneira.

Posner, R. (1973). An economic approach to legal procedure and judicial administration. Journal of Legal Studies, 2(2), 399-458.

Ramseyer, J. M. (2012). Talent matters: Judicial productivity and speed in Japan. International Review of Law \& Economics, 32(1), 38-48.

Rosa, J., Teixeira, C., \& Pinto, J. S. (2013). Risk factors in e-justice information systems. Government Information Quarterly, 30(3), 241-256.

Rosales-López, V. (2008). Economics of court performance: An empirical analysis. European Journal of Law and Economics, 25(3), 231-251.

Røste, R., \& Miles, I. (2005). Differences between public and private sector innovation. Oslo: Publin/NIFU/STEP.

Revista de Administração e Inovação, São Paulo, v. 11, n.2, p. 321-344, abr./jun. 2014. 
Sadek, M. T. (2004). Judiciário: Mudanças e reformas. Estudos Avançados, 18(51), 79-101.

Sadek, M. T., \& Rogério, A. (1994). A crise do Judiciário e a visão dos juízes. Revista USP, (21), 3445.

Saman, W. S. W. M., \& Haider, A. (2013). E-Shariah in Malaysia: Technology adoption within justice system. Transforming Government: People, Process and Policy, 7(2), 256-276.

Schneider, M. R. (2004). Performance management by culture in the national labor relations board's division of judges and the German labor courts of appeal. Journal of Public Administration Research and Theory, 14(1), 19-32.

Schneider, M. R. (2005). Judicial career incentives and court performance: An empirical study of the German Labour Courts of Appeal. European Journal of Law and Economics, 20(2), 127-144.

Schumpeter, J. A. (1982). A teoria do desenvolvimento econômico. São Paulo: Abril Cultural.

Schumpeter, J. A. (2008). Capitalism, socialism and democracy. New York: Harper Perennial Modern Thought.

Sevigny, E. L., Pollack, H. A., \& Reuter, P. (2013). Can drug courts help to reduce prison and jail populations? The Annals of the American Academy of Political and Social Science, 647(1), 190212.

Simon, H. A. (1966). O critério de eficiência. In D. Waldo (Ed.), Problemas e aspectos da administração pública (pp. 518-531). São Paulo: Pioneira.

Smyth, R., \& Bhattacharia, M. (2003). How fast do old judges slow down?: A life cycle study of aging and productivity in the Federal Court of Australia. International Review of Law \& Economics, 23(2), 141-164.

Swanson. (2007). The dynamics of specific and diffuse support for the U.S. Supreme Court: A panel study. The Social Science Journal, 44(4), 645-663.

Tate, C. N., \& Haynie, S. L. (1993). Authoritarianism and the functions of courts: A time series analysis of the Philippine Supreme Court, 1961-1987. Law and Society Review, 27(4), 707-740.

Trawver, K. R., \& Rhoades, S. L. (2012). Homesteading a pioneer mental health court: A judicial perspective from the last frontier. American Behavioral Scientist, 57(2), 174-188.

Tulkens, H. (1993). On FDH efficiency analysis: Some methodological issues and applications to retail banking, courts, and urban transit. The Journal of Productivity Analysis, 4(1), 183-210.

Velicogna, M., Errera, A., \& Derlange, S. (2011). e-Justice in France: The e-Barreau experience. Utrecht Law Review, 7(1), 163-187.

Velicogna, M., Errera, A., \& Derlange, S. (2013). Building e-justice in Continental Europe: The TéléRecours experience in France. Utrecht Law Review, 9(1), 38-59.

Vidal, J. B. i, \& Leaver, C. (2011). Are tenured judges insulated from political pressure? Journal of Public Economics, 95(7), 570-586.

Revista de Administração e Inovação, São Paulo, v. 11, n.2, p. 321-344, abr./jun. 2014. 
Voigt, S. (2009). The effects of lay participation in courts - A cross-country analysis. European Journal of Political Economy, 25(3), 327-339.

Voigt, S. (2012). On the optimal number of courts. International Review of Law \& Economics, 32(1), 49-62.

Waldo, D. (1948). The administrative state: A study of the political theory of american public administration. New York: The Ronald Press Company.

Waye, V. (2003). Judicial fact-finding: Trial by judge alone in serious criminal cases. Melbourne University Law Review, 27(2), 423-457.

Willoughby, W. F. (1929). Principles of judicial administration. Washington: The Brookings Institution.

Wilson, W. (1887). The study of administration. Political Science Quarterly, 2(2), 197-222.

Wu, K. X. (2013). Experiences that Count: A comparative study of the ICTY and SCSL in shaping the image of justice. Utrecht Law Review, 9(1), 60-77.

Yeung, L. L., \& Azevedo, P. F. (2011). Measuring efficiency of Brazilian courts with data envelopment analysis (DEA). Journal of Management Mathematics, 22, 343-356.

Yunker, J. A. (1983). The effect of decision time and argument complexity on legal judgment. International Review of Law \& Economics, 3(2), 161-178. 


\title{
INNOVATION AND PERFORMANCE IN JUDICIAL MANAGEMENT: UNCOVERING CONCEPTUAL AND METHODOLOGICAL GAPS
}

\begin{abstract}
Innovation - a new product or technological, organizational or marketing process - presupposes performance improvement. In public services, especially in the Judiciary, innovation appears as a more complex and multifaceted phenomenon. The literature could be better explored from the innovation perspective. The aim of this paper is to build an articulate discussion on the constructs innovation and performance in the Judiciary in order to show their relationships, conceptual and methodological gaps and propose a research agenda. It is analyzed the state of the art of these concepts, which allowed classifying topics in three dimensions: organizational-managerial, political-legal and technological. The results indicate that: (i) there are similar variables on both constructs, (ii) the literature on performance is more voluminous and consolidated, (iii) important for organizational-managerial dimension contributions were seen in studies of law and economics perspective, (iv) major intersections were found in the technological dimension, specifically amongst innovation, performance and Information and Communication Technologies - ICTs. A research agenda is proposed aiming to fulfill identified gaps.
\end{abstract}

Keywords: Innovation; Performance; Judiciary; Public Management.

Data do recebimento do artigo: 20/01/2014

Data do aceite de publicação: 16/05/2014

Revista de Administração e Inovação, São Paulo, v. 11, n.2, p. 321-344, abr./jun. 2014. 